\title{
Design and Development of a PC based ECG Equipment
}

\author{
Abdullah Al-Amin, A K M Bodiuzzaman, Ahamad Imtiaz Khan, K Siddique-e Rabbani \\ Department of Biomedical Physics \& Technology, University of Dhaka, Dhaka, Bangladesh \\ email: srinku2004@yahoo.com, rabbani@univdhaka.edu
}

Received: 13 August 2013, Accepted: 15 December 2013

\begin{abstract}
ECG is very important for diagnosis of cardiac disorders but most ECG equipment come from developed countries with very costly procurement and maintenance. Therefore, unless the technology is developed indigenously, common people in countries like Bangladesh will not get the benefits of modern healthcare technology. With the advent of personal computers (PC) at low cost, a PC based ECG equipment is also worth developing since many people already own a PC, thereby reducing the overall cost. With this view a PC based ECG equipment was designed and developed from the basics. An attempt was made to utilize maximum components and spares available in the local market. The main unit is based on a low cost but quality instrumentation amplifier IC with high common mode rejection ratio. Active filters were designed using common operational amplifier ICs. Right leg drive circuitry to reduce $50 \mathrm{~Hz}$ noise of mains ac was kept as an option. Analog signal switching circuitry was used to obtain 12 Lead configurations, which were programme controlled, through graphical user interface. To achieve electrical isolation between patient and the mains ac, an analogue optical isolation circuit was designed and developed. Finally the analogue signal was converted to digital data and interfaced to a personal computer through its USB port using a microcontroller. The required power is obtained by USB port 5V supply. A medical grade isolated dc to dc converter was designed to power the patient side circuitry. Proper patient safety issues were followed in the design. Calibration was performed to make the equipment as accurate as possible. Outputs from several subjects were compared with that of reliable commercial equipment and were found to be satisfactory by cardiac experts.
\end{abstract}

\section{Introduction}

ECG or Electrocardiogram is the electrical picture of the human heart and an ECG equipment is needed to pick up and display these electrical signals from the body of a human subject. An ECG equipment is therefore, a very essential item in any healthcare centre, whether in the rich countries of the First World or the poor countries of the Third World. However, manufacture and commercialization of such equipment only by the First World have caused an undesirable situation; almost $80 \%$ of global population living in the Third World is not getting the benefits of such modern equipment of healthcare. ECG equipment exemplifies this statement; it was invented more than a hundred years back but the majority of the global population living in the Third World still do not get benefit of this equipment (Rabbani 1995a, Rabbani 1995b). This is mainly because of high cost of procurement, maintenance and repair, and remoteness of manufacturer from user. Most of the modern medical equipment use microcontrollers with firmware controlled design, and for trade secrecy, the manufacturers do not provide technical details or proper repair manuals, thus making local repairs almost impossible. In many rural hospitals in Third World countries like Bangladesh, either there is no ECG equipment, or if there is one, it is probably out of order (Rabbani et al 2011). Therefore, it is necessary that such equipment are designed and developed indigenously in each country for local manufacture and distribution. With this view a computerized ECG equipment has been designed and developed from the basics at the department of Biomedical Physics \& Technology, University of Dhaka.

Although ECG is a standard equipment, much of the detailed designing is performed by the industry, not by the academia. Therefore, detailed technical information on its design is not available in the 
literature. For example, a detailed circuitry depicting the input network needed to achieve the 12 lead diagnostic ECG could not be found after a thorough search over the internet although basics of the amplifier circuitry was available. Therefore, we had to develop this front end circuitry based on the basic scientific understanding of the problem. The ECG circuitry was optimized to work with a personal computer or a Laptop computer and therefore, has an approach not common with traditional stand-alone instruments having built-in printers for graphical outputs. Besides, the electrical isolation circuitry for the signal was designed using a non-conventional approach to keep things as simple as possible but giving an acceptable quality. This paper gives a detailed description of the design developed in the present work. Firstly, basic information on the source of the 12 lead ECG signals from the human body is provided in order to relate the design of the circuitry that follows.

The function of pumping blood throughout the body requires contraction and relaxation of heart muscles which is produced by electrical activity in the form of depolarization and repolarization of muscle fibers. The electric field generated due to the charge separation around the muscle fibers of heart is propagated through the composite aqueous dielectric and conducting medium of the human body and is available on the surface of the human body in the form of a time varying voltage signal, when measured between a pair of suitable points and displayed in time using appropriate apparatus. This time varying voltage signal is called the ECG. ECG signals for different combination of points on the body are called Lead positions. There are 12 different clinically standard Lead positions for which 10 skin surface electrodes are attached to various parts of the body as shown in Figure 1a. 12 different combinations of these electrodes produce the clinically standard 12 Lead ECG trace (Maryland 2009, Nottingham 2009). The Leads can be divided into two categories Bipolar and Unipolar. Bipolar Leads have one negative and one positive pole. Unipolar Leads also has negative and positive pole but the negative pole is obtained from a combination of more than one electrodes.

Electrodes placed in the limbs are Right Arm (RA), Left Arm (LA) and Left Leg (LL) as shown in Figure.1a. Three bipolar classical limb leads are obtained as follows, Lead I: voltage difference between positive point LA and negative point RA, Lead II: voltage difference between positive point LL and negative point RA and Lead III: voltage difference between positive point LL and negative point LA.

Since current due to the electrical activity of the heart muscle does not flow through the limbs, it circulates within the thorax only, the electrical potentials at the base and at the extremity of a limb is the same with respect to ECG signals. Therefore, ECG potential at the wrist of left arm is the same as that at the base of the arm on the thorax, and similarly for the other limbs. Therefore, the position of the limb electrodes can also be shown on the thorax as shown using the corresponding coloured circles in Figure. 1a.

Slightly shifting the position of the LL electrode upwards and towards the centre, electrodes placed on the thorax as Right Arm (RA), Left Arm (LA) and Left Leg (LL) can be made to form an equilateral triangle known as 'Einthoven's Triangle' (Brown et al), also shown superimposed in Figure 1a and shown separately in Figure. 1b. The three classical limb leads effectively produce views of the heart's electrical activity in the directions shown by the corresponding arrows.

These three limb electrode can also produce the three unipolar Augmented Leads as shown by the long arrows in Figure 1b, aVR: voltage difference between positive point RA and negative point as the combination of LA and LL; aVL: voltage difference between positive point LA and negative point as the combination of RA and LL; aVF: voltage difference between positive point LL and negative 
point as the combination of RA and LA. The combination of two electrodes means simply average potential or potential at the mid-point between two electrodes inside the body.

These 3 classical limb leads and the 3 augmented leads together provide views of the electrical activity of the heart in the frontal plane at angles separated by $30^{\circ}$ as shown schematically in Figure. $1 \mathrm{c}$.

In the transverse or horizontal plane (with reference to a standing person), six other unipolar leads are called Precordial Leads: V1, V2, V3, V4, V5 and V6. For these leads six electrodes are placed directly on the chest (Figure 1a) and are the positive points. The negative point for all these six Leads is the Wilsons Central Terminal (Grimnes and Martinsen 2008) which is a combination of the three limb electrodes giving the approximate common or average potential of the body.

The electrical orientation of these precordial Leads in the transverse plane are shown in Figure 2. The Right Leg (RL) electrode is used as common or reference point for differential potential measurement of the positive and negative points of all 12 Leads. The RL is also used for active ac line noise cancellation technique called Right Leg Drive (RLD) (Acharya 2011, Markandey 2010).
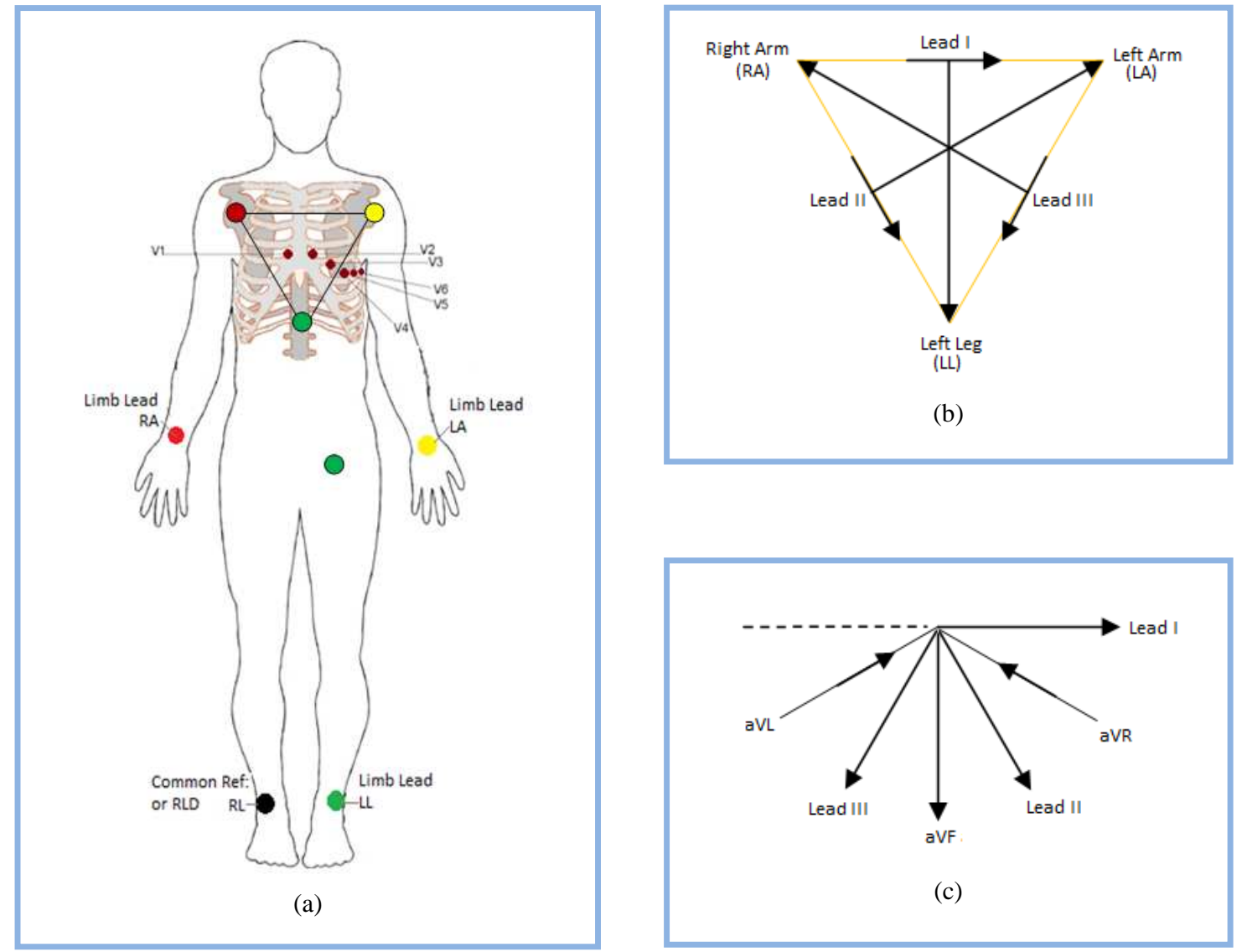

Figure 1: a. Electrode Placement for 12 Lead ECG, Einthoven's Triangle shown superimposed, b. Classical and Augmented limb Leads in Frontal Plane, c. Effective direction of view for heart's electrical activity. 
The shape of the ECG signals for different Leads effectively reveal the presence of any heart disorders by calculating the Cardiac Vector (Brown et al), size and position of the heart chambers, heart-beats, etc.

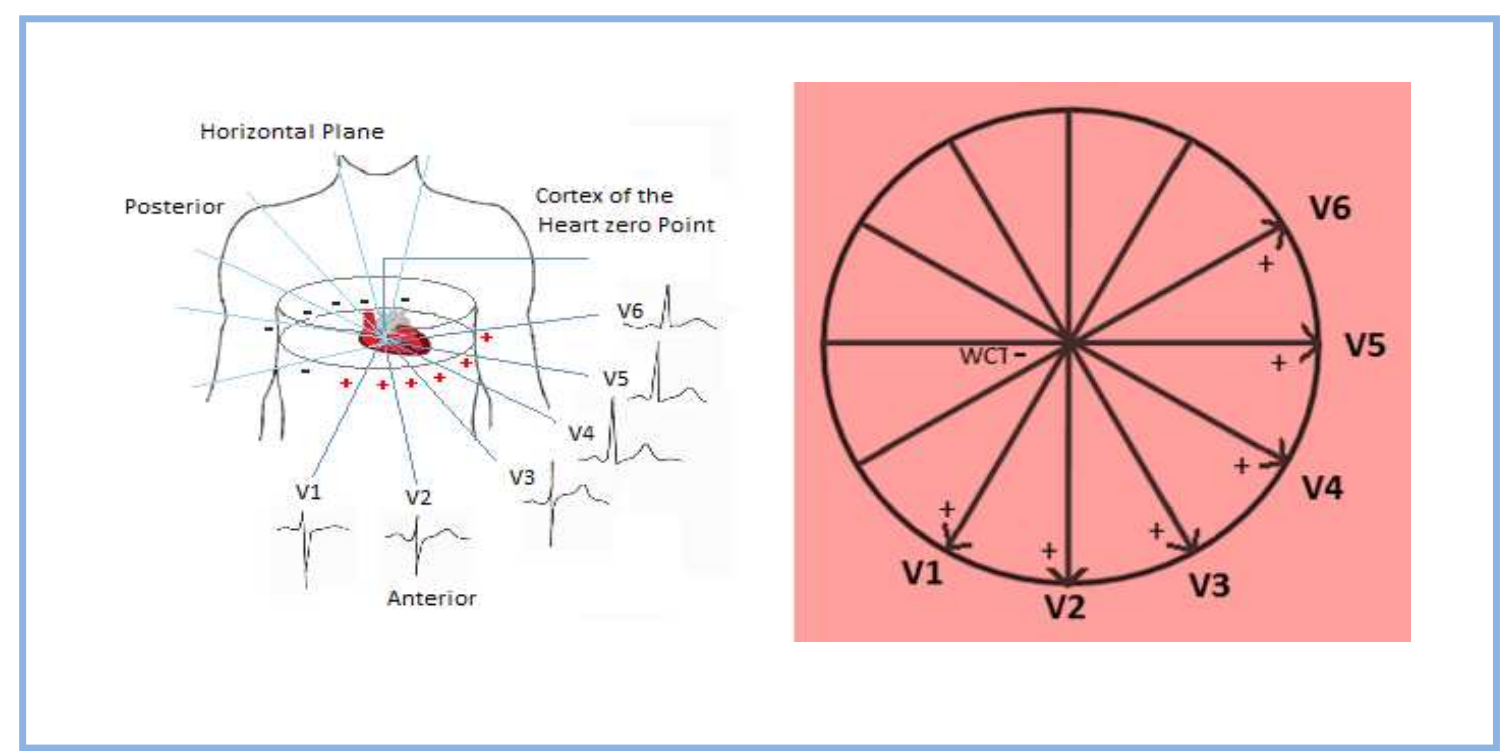

Figure 2: Precordial Leads and the directions of activity in the transverse (horizontal) Plane.

\section{Methods}

\subsection{Basic considerations}

The PC based 12 Lead diagnostic ECG system with data acquisition, storage and display that has been developed in our laboratory will provide easy access to repair or replacement of different parts of the whole system, large storage space for patient history, facility for post processing of data and real time diagnosis by expert doctor for remote patients through telemedicine etc. (Rabbani et al 2011). The ECG equipment will communicate with a PC through its USB port and will be powered from the PC through the same port as well, so that no external power will be required to drive the ECG equipment. This section will give a detailed overview of the design aspects of the ECG amplifier including the differential bio-electric signal measurement, input analogue signal switching for different Leads, data acquisition, electrical isolation, $\mathrm{PC}$ interface, and the challenges that have been overcome to achieve the above.

Typical voltage amplitudes of ECG signals range between $1 \mathrm{mV}$ and $3 \mathrm{mV}$ and has a frequency band of about $0.5 \mathrm{~Hz}$ to $100 \mathrm{~Hz}$. The low voltage signals need to be amplified in a noise free condition before these can be displayed graphically or converted to digital data. In a typical building with mains ac power, the $50 \mathrm{~Hz}$ ac line interference in human body is usually much larger (10 to 20 times). It will override the ECG signal unless this $50 \mathrm{~Hz}$ noise is eliminated or minimized to a very small level, typically less than one hundredth of the ECG voltage. Since this noise frequency falls within the desired signal range, it cannot be eliminated easily using a filter. Fortunately, this $50 \mathrm{~Hz}$ noise voltage is almost the same all over the body, forming a common mode potential and this can be eliminated or minimized through subtraction of two potentials picked up by two electrodes placed across the heart. Therefore this needs a special type of amplifier configuration known as differential amplifier which 
has two inputs with the output being proportional to the difference of the input potentials. All the potentials are measured with respect to a common terminal (in practice RL). A special configuration of the differential amplifier is called the Instrumentation Amplifier (ins-amp) which is conventionally used in an ECG amplifier. It has a high Common Mode Rejection Ratio (CMRR), about $80 \mathrm{~dB}$ or more, and the $50 \mathrm{~Hz}$ signal can be reduced significantly with respect to the desired ECG signal. The ins-amp also minimises the voltage drop across the contact impedances of the skin electrodes, typically 10Kohms at low frequencies, to a negligible level (Rabbani et al 2011).

The measurement of classical bipolar limb leads (I, II \& III) is straightforward as mentioned before. For the Augmented and Precordial leads, combination resistive networks are used as shown in Figure 3 to get the negative points. For the Augmented Leads two equal valued resistors $\mathrm{R}$ between two limb electrode forms a voltage divider network providing the average mid-point potential which is used as the negative point input for the instrumentation amplifier; the positive input being the other limb electrode (Figure 3a). For the Precordial Leads, three equal valued resistors $\mathrm{R}$ in a $\mathrm{Y}$ network produces the Wilsons Central Terminal (Figure $3 \mathrm{~b}$ ) producing the average body potential, which is used as the negative point input for the instrumentation amplifier for all the six Leads, the positive inputs being the six chest electrode (Figure 1a \& c). It needs to be mentioned that in order for this scheme to work the values of $\mathrm{R}$ should be much greater than the electrode contact impedances so that the midpoints defined by the resistor chains are not too far away from the corresponding real midpoints that they intend to represent.

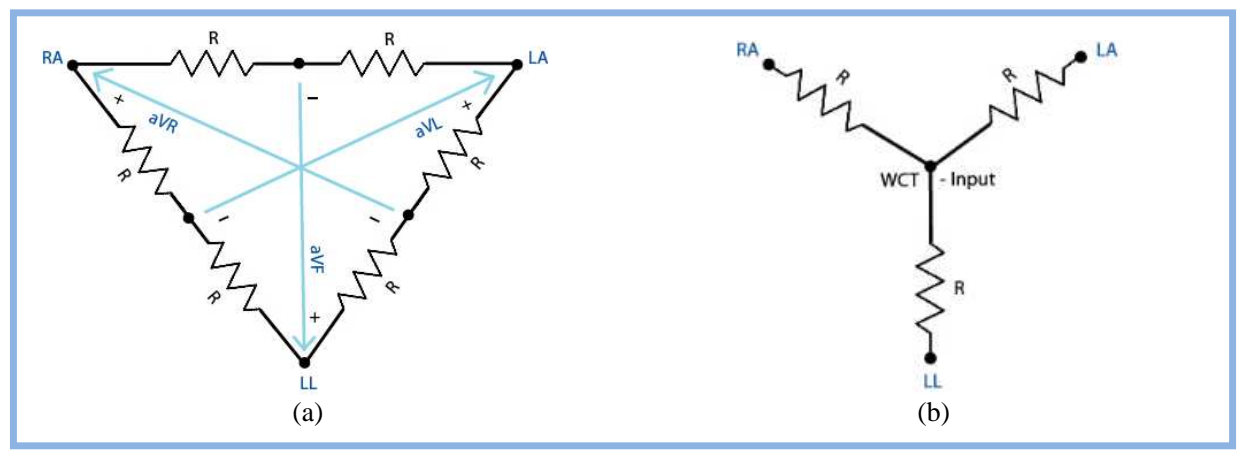

Figure 3: Resistor Network for a. Augmented Leads, b. Precordial Leads

As described earlier, the 12 standard combinations of ECG potentials are obtained using 10 electrodes on the body, which need to be connected and switched in different ways to different resistor networks and the amplifier that follows. The network switching is more complex in the design of single channel ECG equipment since it uses only a single instrumentation amplifier to obtain all the 12 lead signals in sequence. This is also the target in the present design in order to reduce cost. Of course this has the advantage that all the leads go through the same amplifier gain maintaining their relative magnitudes, which may not be maintained accurately if different amplifiers are used for different lead signals in parallel. Thus both the positive and negative inputs of the instrumentation amplifier at the input stage need a network of resistors switched to the 10 body electrodes appropriately. Obviously, this switching will be performed for analogue signals, and one has to take care that the sizes and shapes of the signals are maintained. The total input resistance seen by the two inputs of the instrumentation amplifier should be closely matched, otherwise voltage drop across the input resistors due to noise sources will contribute to a differential noise potential resulting in increased noise in the ECG (Rabbani et al 2011). This requires careful planning and calculation in the choice of the resistor 
network. In the present design all the switching of the input resistor network is controlled by software, known as 'program control' from the computer which needs specialized analogue switch ICs.

Patient safety is major concern for medical equipment. A current of about $50 \mu \mathrm{A}$ of mains ac at $50 \mathrm{~Hz}$ directly flowing through the heart muscle can be fatal, and is known as 'microshock'. As a comparison, the threshold of fatal current is more than a thousand times higher (about 75mA) for normal shock where contact with the mains ac line occurs through skin surface. Microshock is particularly relevant to patients in hospitals who are under trial with external pacemakers with an external catheter connecting directly to the heart muscle. Normal transformers used to isolate mains line ac from low voltage ends where a person can touch electrical contacts as in normal domestic or industrial appliances are not adequate to prevent microshock. Therefore, special electrical isolation must be provided in ECG equipment to reduce the leakage current at $50 \mathrm{~Hz}$ to levels much below $50 \mu \mathrm{A}$.

Since the PC is normally powered by mains supply, the ECG device circuit must be divided into electrically isolated (from mains ac) patient side and non-isolated PC side (Rabbani et al 2011). Both the power and the signal need to be electrically isolated. In the present design, since the controls for the analogue switches to connect the different patient leads to the ins-amp come from the mains connected PC, these control paths need to be electrically isolated too. We have designed and fabricated a medical grade isolated dc to dc converter for supplying power to the isolated side. Here low voltage dc for this converter is obtained from USB power of the PC as mentioned before. For the isolation of the ECG signal and the digital control signals, we have designed and fabricated appropriate optical isolation circuitry using opto-couplers. A safety network of current limiting resistors have been implemented at the input side, just after the electrodes, to limit dc current to the patient to a safe value in case of a severe fault of the input circuitry. Moreover, neon and clamping diodes have been incorporated at the input circuitry to save the ECG amplifier from high voltage spikes and large short duration pulses created by Cardiac defibrillators.

\subsection{Hardware design}

The whole ECG equipment design is explained with the help of Figure.4. The first stage is the resistive network as mentioned before together with the analogue switching circuitry for lead selection. This is achieved with the help of a shift register IC (74HC164), which controls the analog switching of the lead selection, and three different digitally controlled analog signal switch ICs (4051, $4052,4053)$. The circuit details are given later.

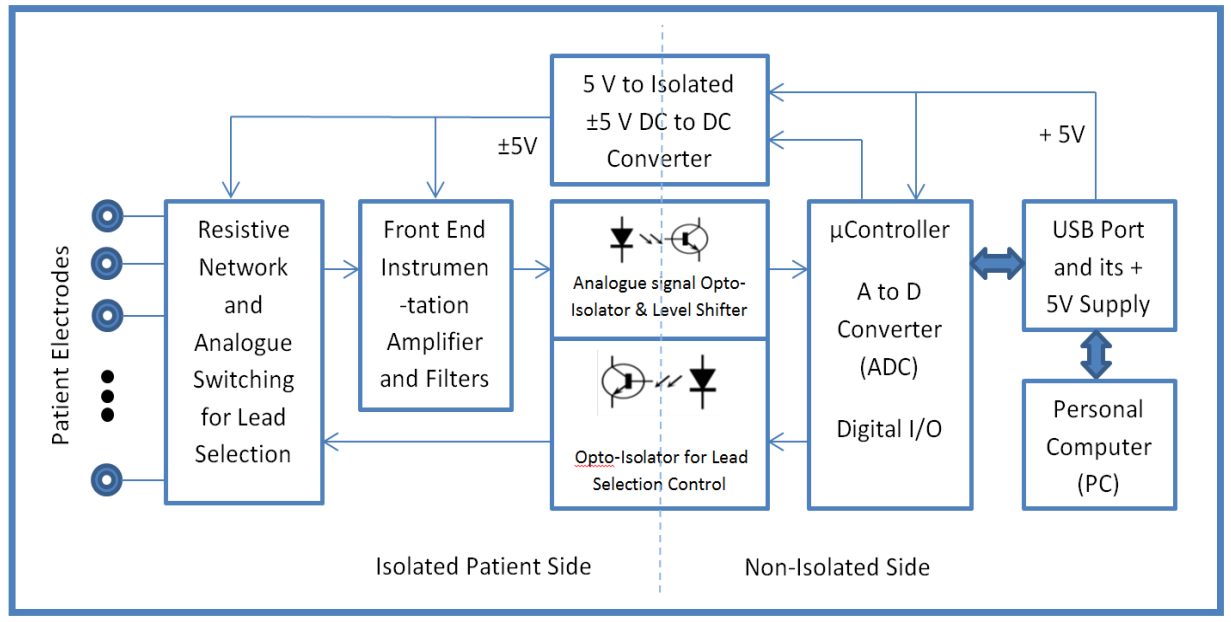

Figure 4: Block Diagram of Cqpputerized 12 Lead ECG System 
The second stage in the ECG equipment is a differential amplifier stage, implemented in the present design using an instrumentation amplifier IC (LT1167). Following this an active band pass filter within the ECG signal band is constructed using standard operational amplifiers (TL072), followed by a second stage amplifier to match precise gain requirements. Since the ECG signal has components between $0.5 \mathrm{~Hz}$ and $100 \mathrm{~Hz}$, with more energy at the lower frequencies, a band pass filter with cut off frequencies of about $0.05 \mathrm{~Hz}$ and $200 \mathrm{~Hz}$ are required to avoid phase distortion in the signal.

A Right Leg Drive (RLD) circuit is also implemented for active cancellation of common mode noise. However, a provision has been made to switch off the RLD when the RL electrode is used as a simple neutral or common terminal, again through software controlled switching.

The third stage is the electrical isolation stage where the patient connected circuitry on the left side is electrically isolated from the mains connected circuitry on the right hand side. The isolation consists of three parts, one for power isolation, one for analogue signal isolation and the other for isolating the digital control signals required for input network switching as mentioned before.

A microcontroller with integrated Analog to Digital Convertor (ADC) is used at the non-isolated side which is the main unit for data acquisition, communication and control of the ECG device. It performs multiple functions through program control providing i) DC shift of non-isolated analog signal circuit (by either $2.5 \mathrm{~V}$ or $1.28 \mathrm{~V}$ ) for two different ADC references $(5 \mathrm{~V}$ or $2.56 \mathrm{~V}$ ) required by our design, ii) acquisition of the optically isolated analog signal, iii) control signals for the input lead selection, iv) pulses for the dc to dc converter which is also used for negative supply (-5 volt) generation for the non-isolated analog signal section (performed through a capacitor charge pump) and v) USB interface for ECG data transfer and communication with PC.
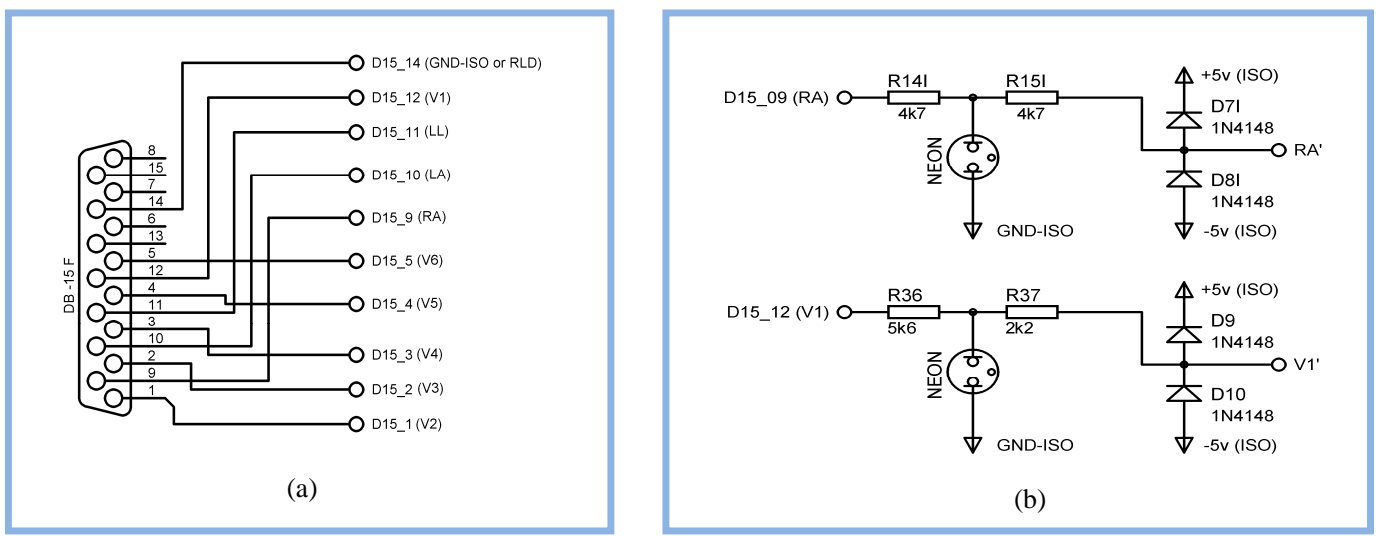

Figure 5: a. Patient electrode connection diagram through DB-15 connector, b. Input network for patient safety and formation of resistor network for the electrode leads.

For the present design a DB-15 connector was used for patient lead, as employed by most commercial 12 lead-ECG equipment. This provides compatibility with other devices and easy replacement. Figure 5a shows the pin connections of the DB-15 connector for ECG equipment. A passive input circuit follows the patient connections and a pair is shown in Figure 5b. The series resistors are used for both leakage current limitation (for patient safety) and formation of a passive low pass filter (fc at $100 \mathrm{~Hz}$ ). The latter is achieved in conjunction with the capacitor and part of a resistor network that follows for Augmented and Precordial Leads. The neon lamp is used for protection from relatively slow high voltage pulses such as that from defibrillators while the low pass filter mentioned above reduces fast 
spikes. The clamping diodes clamp the input to maximum of $\pm(5+0.3) \mathrm{V}$ for protection of both the patient and the input circuitry of the instrumentation amplifier. In Figure $5 \mathrm{~b}$ only two circuits are shown for each of the inputs of limb (RA, LA, LL) and chest (V1 to V6) electrodes; they differ only by the values of the resistors which are necessary for different resistive networks of Augmented and Precordial leads. The RL input is directly connected to the isolated ground or RLD as chosen.

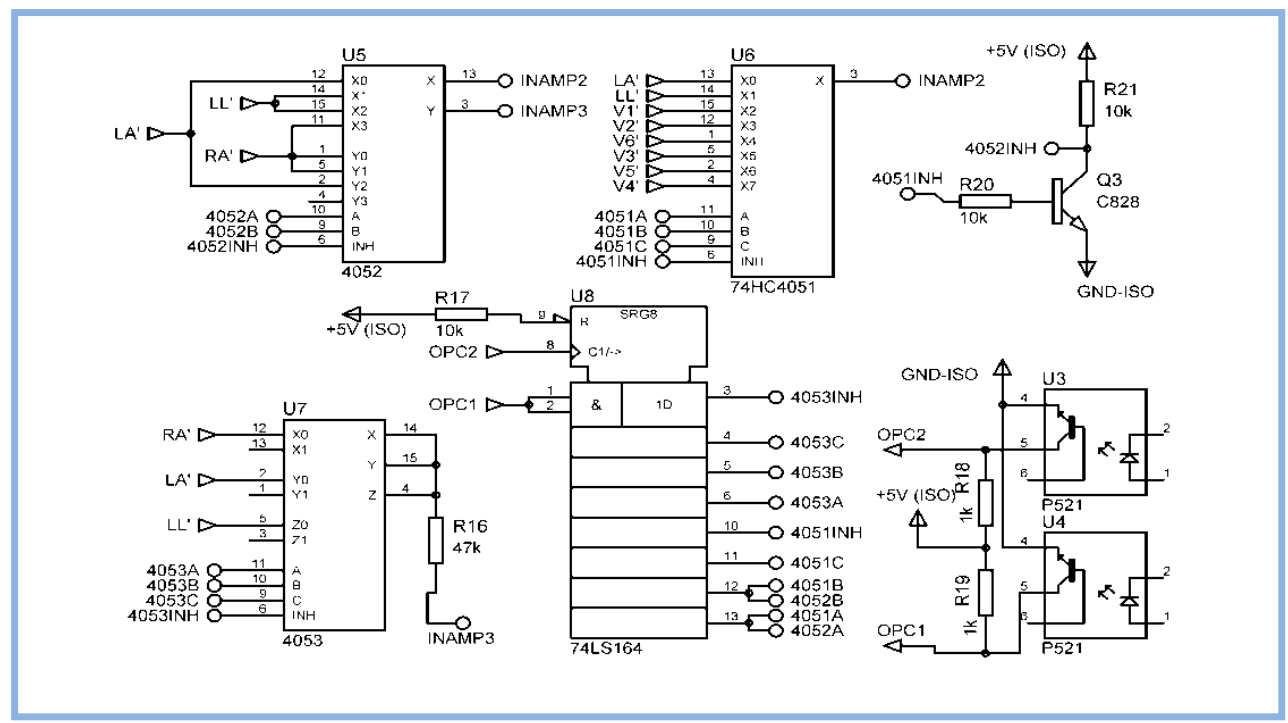

Figure 6: Analog switching circuits for different leads and the isolated segments of the optical isolation circuits

Together with the input resistors, the analog switching circuits as shown in Figure 6 provide different combination of resistor networks for all the 12 Leads of ECG. The classical limb Leads (I, II, III) are bipolar so when switched for these Leads, no combination of extra resistor network is needed i.e. the instrumentation amplifier inputs are connected with the limb electrodes only through the series resistors as shown in Figure. 5b. For the Augmented (aVR, aVL, aVF) and Precordial Leads (V1 to V6), switching is performed in such a manner that the input resistors of the limb electrodes form the appropriate resistor networks to the inverting input of the instrumentation amplifier as described earlier (Figure 2). The non-inverting input is connected to the appropriate limb or chest electrodes through the passive resistor network as shown in Figure. 5b.

The Analog Front End (AFE) circuits shown in Figure 7 are composed of an instrumentation amplifier IC (LT 1167) whose gain is set to 9. The gain is kept low to avoid saturation of the amplifier if a high noise voltage is generated at the input leads. At this gain it has a CMRR of about $100 \mathrm{~dB}$ This is followed by a low pass active filter (U10:B) with cut-off frequency of $160 \mathrm{~Hz}$, then a high pass active filter (U10:A) with cut-off frequency of $0.03 \mathrm{~Hz}$ forming the band pass filter required for ECG signal. The high pass filter has long time constant $(5 \mathrm{sec}$ ), so whenever a Lead is changed, it takes long time to settle the new signal to the baseline. Therefore a transistor (Q4) is used to reset the filter through fast discharge of the capacitor every time a Lead is changed. The second stage amplifier was given a gain of 33 .

The amplified ECG signal is then optically isolated through analog signal isolation which is very simple and effective for this application, originally proposed and developed in our laboratory earlier (Rabbani et al 2011). For this isolator, the light intensity of the LED in an optocoupler (U2) is modulated in proportion to the input ac signal. The LED of the optocoupler has to be dc biased which 
is achieved using a current mode summing amplifier (U11:B). Since the output ac variation at the collector end of the non-isolated side of the optocoupler only depends on the variation of the LED drive current due to the input ac signal, the degradation of optical component does not affect the analog signal response.

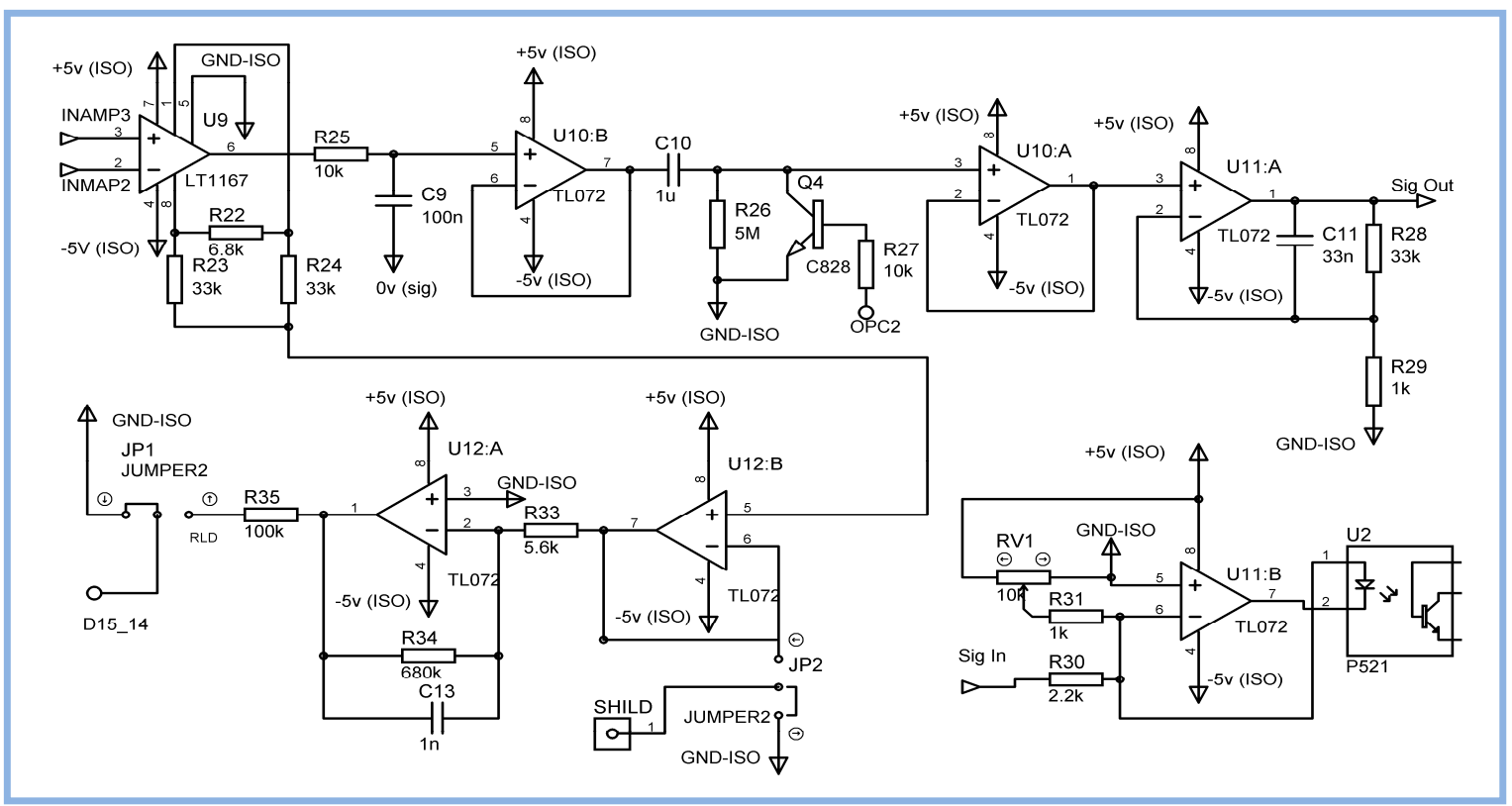

Figure 7: Analog Front End with RLD and Optical Signal Isolator

The RLD circuit (composed of U12:A\&B) takes the common mode noise (ac 50Hz) from the midpoint of the first differential output section of the instrumentation amplifier as input, amplifies with reversed phase and then drives the common lead (RL) of the body to cancel out $50 \mathrm{~Hz}$ interference. This effectively achieves a higher noise cancellation than that from a simple ins-amp. Furthermore, a provision has been kept in which the a voltage equal to the input signal in the same phase drives the metallic shield of the isolated circuit and the electrode cable shield (SD) for reduction of capacitive coupling of $50 \mathrm{~Hz}$ interference. Either the RLD or the SD is selectable through a jumper (JP1\&2).

Figure 8a shows the Atmel AVR series 8 bit microcontroller ATmga8, which is used for data acquisition, control and PC interface through USB. The work load of this microcontroller was described earlier in this section. Figure $8 \mathrm{~b}$ shows the optical isolators for synchronous serial digital interface using the shift register at the isolated section. Figure $8 \mathrm{c}$ shows the non-isolated side of the analog signal optical isolator. Though level of the dc shift of the collector (U2, pin 5) is controllable at isolated section by RV1 (Fig. 7) to make it suitable for ADC input, the output from the collector is ac coupled and dc shifted through a network utilizing capacitor (C5), resistors (R 5,6,7,8) and transistor (Q2). Two different scales of ECG trace display, $5 \mathrm{~mm} / \mathrm{mV}$ and $10 \mathrm{~mm} / \mathrm{mV}$, were achieved simply through switching two different dc shifts of analog signals for the ADC input, $2.5 \mathrm{~V}$ and $1.28 \mathrm{~V}$, in conjunction with reference voltages of $5 \mathrm{~V}$ and $2.5 \mathrm{~V}$ respectively. This essentially gives an alternative way to double the gain. The dc shift is switched using transistor Q2. When Q2 is off, the dc shift is 2.5 $\mathrm{V}$, which is treated as the midpoint or zero level for ac signals at the ADC input, thus using the whole dynamic range of 0 to 5 volt. When Q2 is on, the dc shift is $1.28 \mathrm{~V}$, which is the midpoint of the ac signal at the ADC input; the dynamic range is then between 0 and $2.56 \mathrm{~V}$. Thus the two settings 
effectively gives two gains, one double the other. The referencing facility available with this particular $\mathrm{ADC}$ has been thus used simply to effect a requirement of doubling the gain.

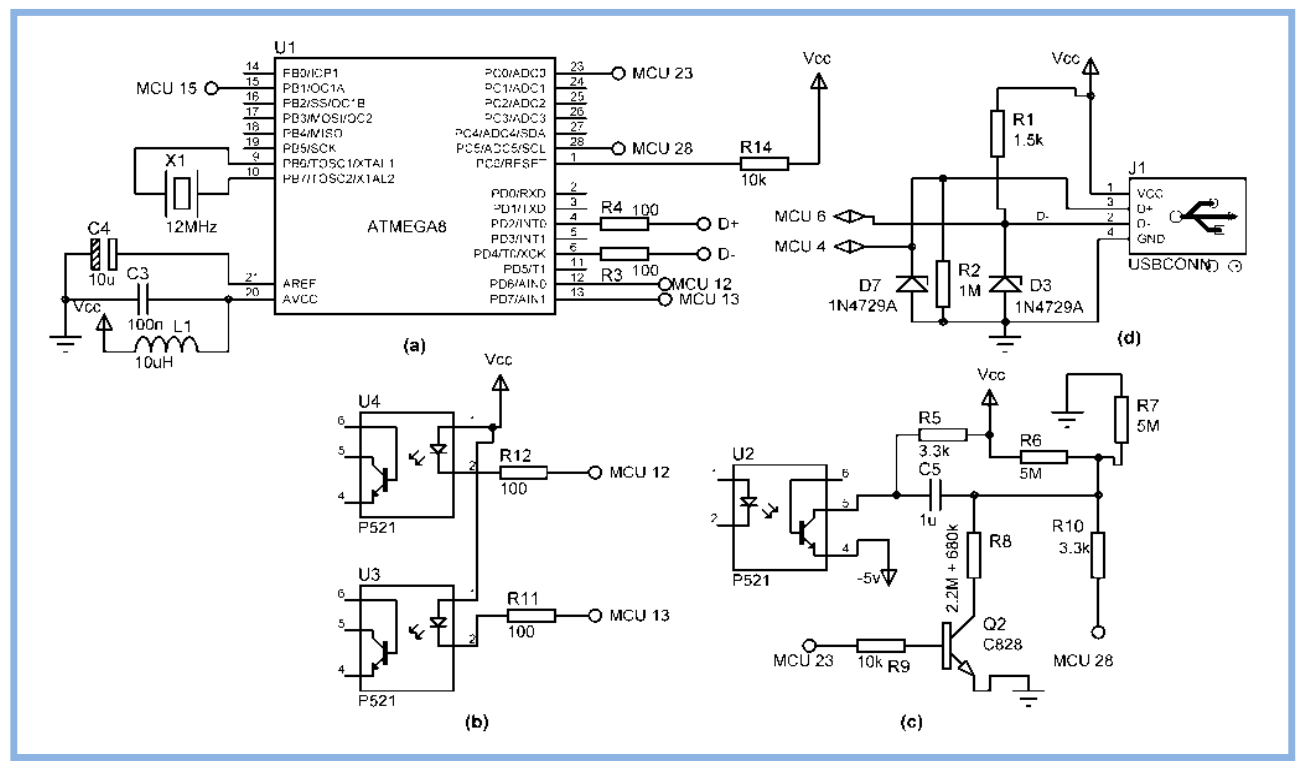

Figure 8: a. Atmel AVR series 8 bit microcontroller ATmga8, b. Optical isolators for synchronous serial digital interface, $\mathrm{c}$. Analog signal optical isolator at the non-isolated side, output of this circuit is connected to microcontrollers ADC input (Pin 28), d. USB connection circuits at the device end.

The output of the phototransistor in the optocoupler (collector of U2) is usually non-linear near the high and low voltage rails, Therefore, to maintain linearity down to $0 \mathrm{~V}$, the phototransistor circuit is operated between $+5 \mathrm{~V}$ and $-5 \mathrm{~V}$ so that $0 \mathrm{~V}$ is much above the lower rail. The optical isolation circuit has a gain of 1.7, providing an overall gain of 510 with reference to the patient input. This gain has

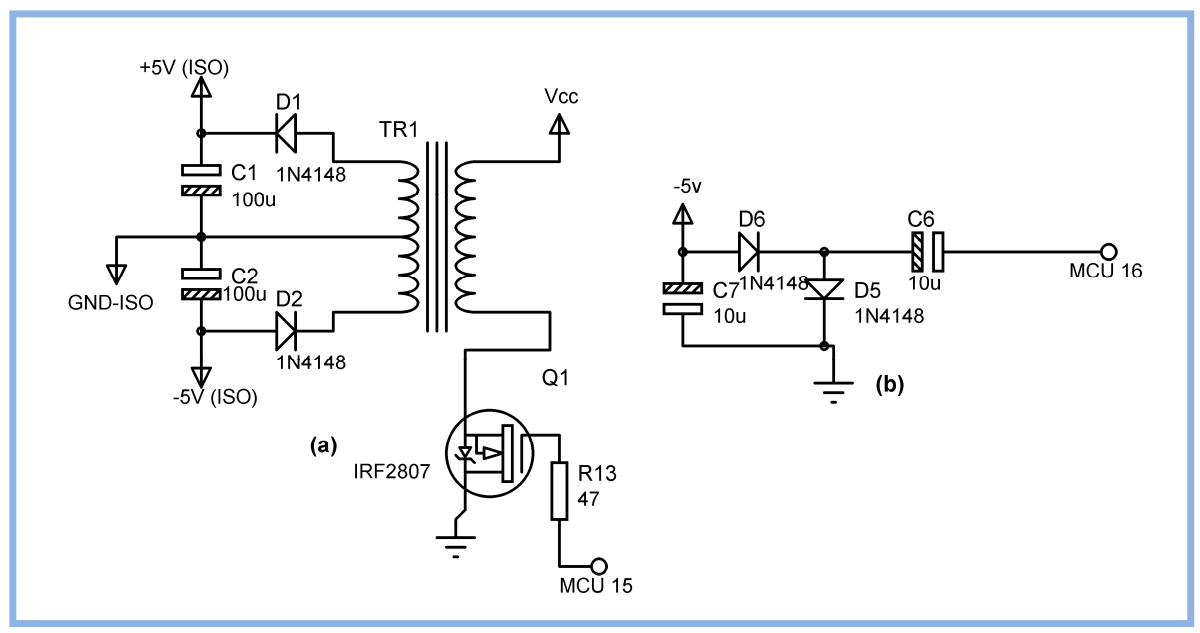

Figure 9: Power Supplies; a. The isolated dc to dc converter, b. Capacitor Charge Pump

been set considering a maximum ECG signal of $\pm 5 \mathrm{mV}$, which will be amplified to $\pm 2.55 \mathrm{~V}$ as the input to the ADC. The largest ECG signal will thus be well within the maximum ADC input voltage of 0 to $5 \mathrm{~V}$ with the midpoint set at $2.56 \mathrm{~V}$, which will correspond to a scale of $10 \mathrm{~mm} / \mathrm{mV}$ on the displayed plot. Figure 8d shows the USB connection circuits at the device (microcontroller) end. The 
3.3 $\mathrm{V}$ zener diodes ensures the maximum signal level of D+ and D- lines, $\mathrm{R} 1$ is used to pull up D- as required by the USB low speed device specification and R2 ensures low level at D+.

The dc to dc converter to obtain isolated $\pm 5 \mathrm{~V}$ supply is shown in Figure 9, which uses a forward topology. A switching signal of $90 \mathrm{KHz}$ is generated by the Pulse Width Modulation (PWM) module of the microcontroller through programming. Since the input 5V (USB power) and output load is fixed, no voltage regulation feedback is used and approximately \pm 5 volt is fixed at the isolated output by calibrating the duty cycle of the switching signal once. The transformer is built on a toroidal type ferrite core. Between the primary and secondary coils approximately $1 \mathrm{~cm}$ of distance is maintained to achieve very low inter-coil capacitance required for patient safety. The non-isolated $-5 \mathrm{~V}$ is also generated by a capacitor charged pump driven by the microcontroller PWM module.

\subsection{Data Acquisition and Computer Interfacing}

The ADC module of the microcontroller is 10 bit successive approximation type. It has two selectable internal references 5 and $2.56 \mathrm{~V}$. After digitally converting the ECG signal the most significant 8 bits are used as the least significant bits are more prone to noise. Besides, data storage and transfer is more efficient if only one byte ( $8 \mathrm{bit}$ ) is used. The two reference of the ADC produces the resolution of 20 and $10 \mathrm{mV}$ per bit for 8 bit conversion respectively (for the two gains). The sampling frequency was chosen at $800 \mathrm{~Hz}$ to match the USB timing and data transfer rate. Since the maximum ECG frequency is $100 \mathrm{~Hz}$, this sampling frequency is adequate.

The computer interface for data acquisition and software device control is performed following USB 1.1 low speed device specification. The microcontroller used is a low cost one and does not have any hardware USB stack. The USB library called V-USB (Atemel 2013b) in the firmware is used for implementation of USB 1.1 low speed standard protocol. The ECG data is transferred to a PC by 'Interrupt in transfer' protocol. The USB interface of the microcontroller can transfer a maximum of 8 bytes every $10 \mathrm{~ms}$ and the host (PC side USB host controller) is programmed to make a request for this transfer very precisely every $10 \mathrm{~ms}$. Therefore, this timing is adopted as a cue for starting ADC conversion. So at every $10 \mathrm{~ms}$ there are 8 bytes of data conversion at equal interval of $1.25 \mathrm{~ms}$ resulting in a sample rate of $800 \mathrm{~Hz}$. The sampling and the data transfer are done simultaneously and are well synchronized. ECG sampling to display has a delay of approximately $10 \mathrm{~ms}$, which is not significant for human perception and this data acquisition can be regarded as real time. The software control of Lead selection and other configuration is achieved by 'Control in and out transfer' protocol.

\subsection{Software}

The PC side software developed in the present work has a graphical user interface (GUI) for ECG display, storage, report generation, device configuration control and online data transfer for telemedicine. It was developed using 'JAVA' platform considering an operating system (OS) independent feature, though only MS Windows version has been tested and used so far. The open source and free interface development environment (IDE) 'Netbeans' (Oracle 2013) was used for development of this software. The software has been developed to have three modes of operation: (a) data acquisition, storage and display, (b) online data transfer, (c) online data receipt, storage and display. For a stand-alone usage of the ECG equipment only the first mode will be used. For a telemedicine system all the three modes are required. The first and second modes are appropriate for a remote centre in this system where the patient data is acquired and sent to an expert centre over internet. The third mode is for the expert centre which will process the received digital data for storage and display. 
A digital notch filter of IIR (Infinite Impulse Response) type was also implemented for optional preprocessing the data to eliminate $50 \mathrm{~Hz}$ line interference. The filter has a sharp stop band between 49 and $51 \mathrm{~Hz}$ and can be turned on or off through program control. Though filtering within the ECG frequency band can deform the signal (Rabbani et al 2011) it is useful for extremely noisy environment where $50 \mathrm{~Hz}$ line interference is very large. The screen shot of the GUI and the generated report are shown in Figure 12 and 13 respectively. The firmware for the microcontroller was developed using free GNU C compiler Winavr (Winavr 2013) with AVR Studio IDE (Atemel 2013a).

\subsection{Calibration}

The supply rail of \pm 5 volt at the isolated section was set by varying the duty cycle of the PWM signal through in circuit programming of the microcontroller. The dc shift of the analog optical isolator output at the non-isolated side was kept close to zero level by adjusting the multi-turn variable resistor VR1 (Fig. 7). It was mentioned earlier that the phototransistor U2 at the non-isolated side was biased at $\pm 5 \mathrm{~V}$ and ac coupled to the input of the ADC. For signal calibration, sinusoidal signals of low frequencies from a generator were optically isolated (to break the ground loop for noise reduction) and attenuated to generate $1 \mathrm{mV}$ peak to peak, and then were fed to the input electrode connectors. The connections were interchanged to make one as input and the other as ground level for each lead, since the generated signal is not differential. For example, for Lead I, it is selected from software so the analog switching is configured for it, then the signal is applied to RA while both LA and RL were kept at signal ground. The software was checked for both $5 \& 10 \mathrm{~mm} / \mathrm{mV}$ scales along the vertical of the plots. Then signal was applied to LA keeping both RA and RL at ground level and repeating the procedure. For Augmented and Precordial leads, appropriate electrodes were shorted together and connected to the inverting input while the active input electrode was connected to the non-inverting input, as described before. Through these measurements fine adjustments of gain was obtained through adjustment of a multiplication factor of the ADC value since the exact gain for different devices will not be same due to tolerance of the components.

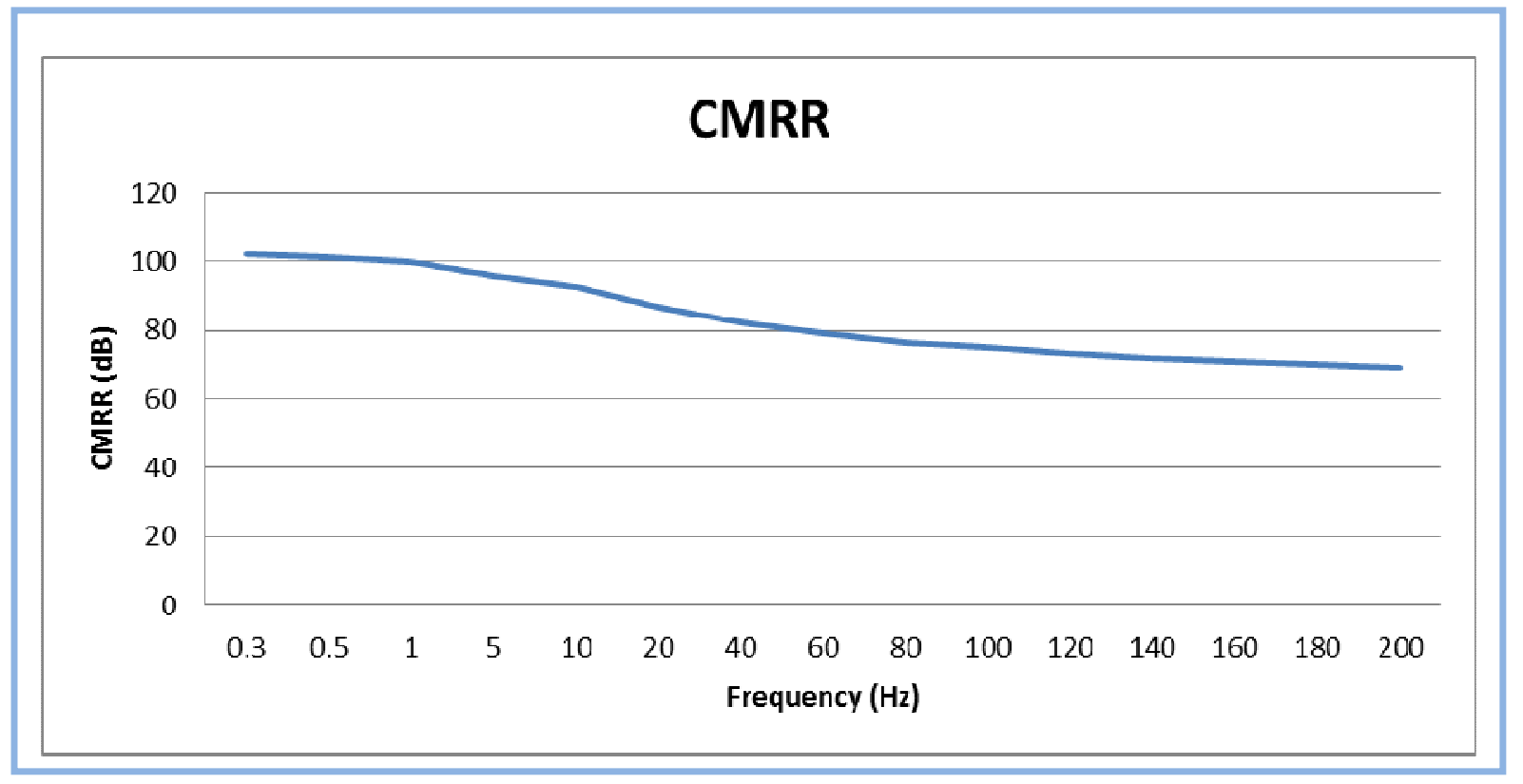

Figure 10: Measured CMRR of the ins-amp as a function of frequency 


\section{Results and Observations}

The CMRR of the instrumentation amplifier was found to be around $100 \mathrm{~dB}$ at low frequencies which matches the manufacturer's data sheet. The measured CMRR as a function of frequency for the insamp is shown in Figure 10. The gains of the different stages amplifiers are well within the expected theoretical values, and the multiplication factor for calibration at software end deviated very little from unity $(\sim 10 \%)$. The measured overall gain of the system as a function of frequency is shown in Figure 11.

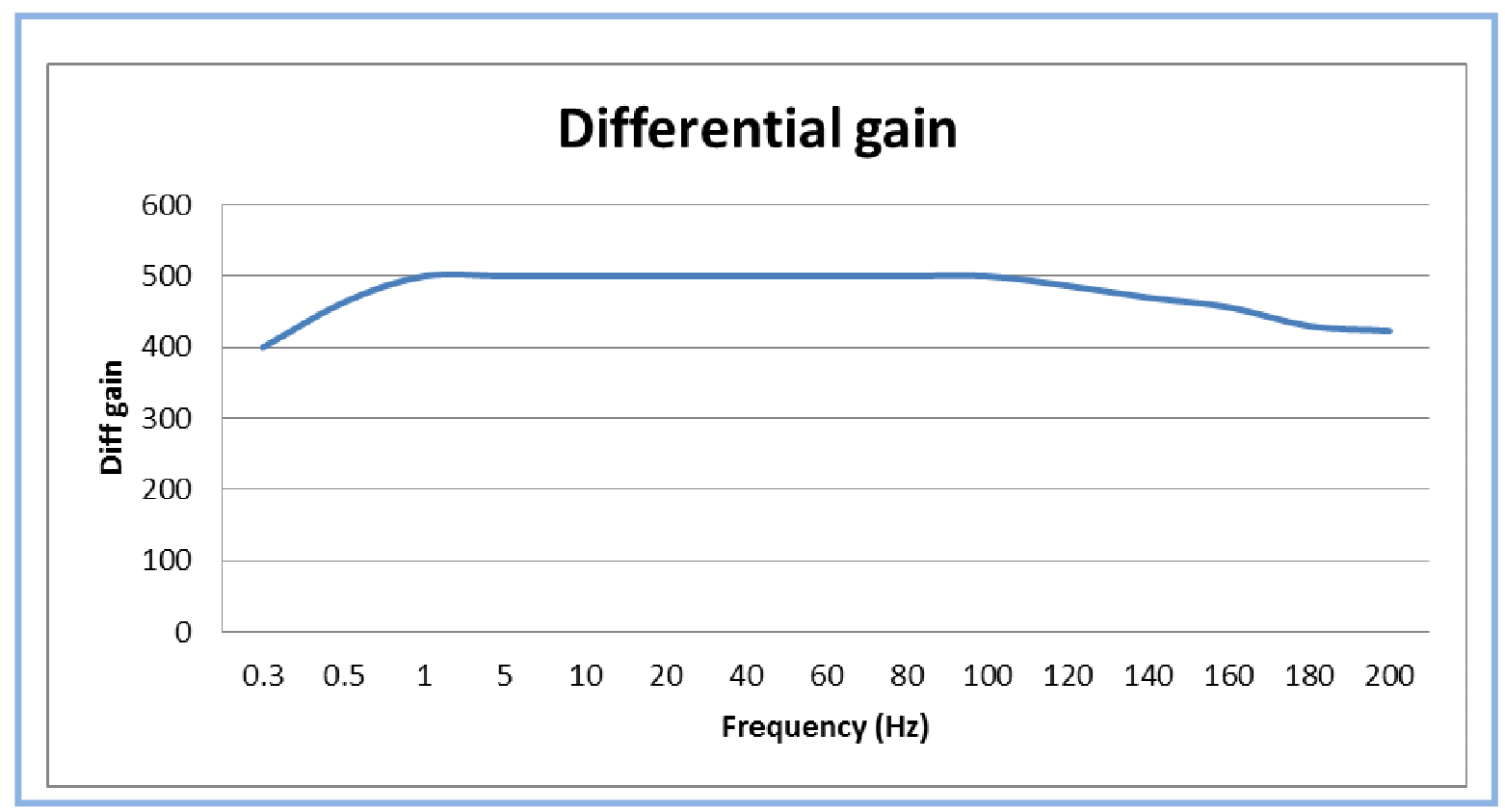

Figure 11: Measured differential gain as a function of frequency

The RLD circuit effectively reduces $50 \mathrm{~Hz}$ noise, but it introduced some high frequency noise with approximately one tenth magnitude of $50 \mathrm{~Hz}$ noise. Therefore, its use was restricted to only locations with high $50 \mathrm{~Hz}$ noise. It is also observed that almost $80 \%$ noise is reduced only using a metallic shield covering the patient side circuitry and using shielded cables for electrodes. No significant difference was observed connecting the shield of the cable to isolated ground or by driving it actively (driven shielded). The digital filter could eliminate $50 \mathrm{~Hz}$ noise even if its magnitude was almost twice as the ECG signal

The switching of various Leads works very well as expected. A large number of real subjects were tested in our laboratory using this equipment and the resulting ECG wave shapes were compared with that obtained using commercially available ECG equipment from reputed manufacturers. The wave shapes and their magnitudes appeared identical visually. Tests performed at a premier cardiology hospital in the country gave very satisfactory results as reported by cardiac experts informally. The screen shot of the software containing the ECG trace of a real subject is shown in Figure 12 and the generated report containing all Leads graph is shown in Figure 13. 


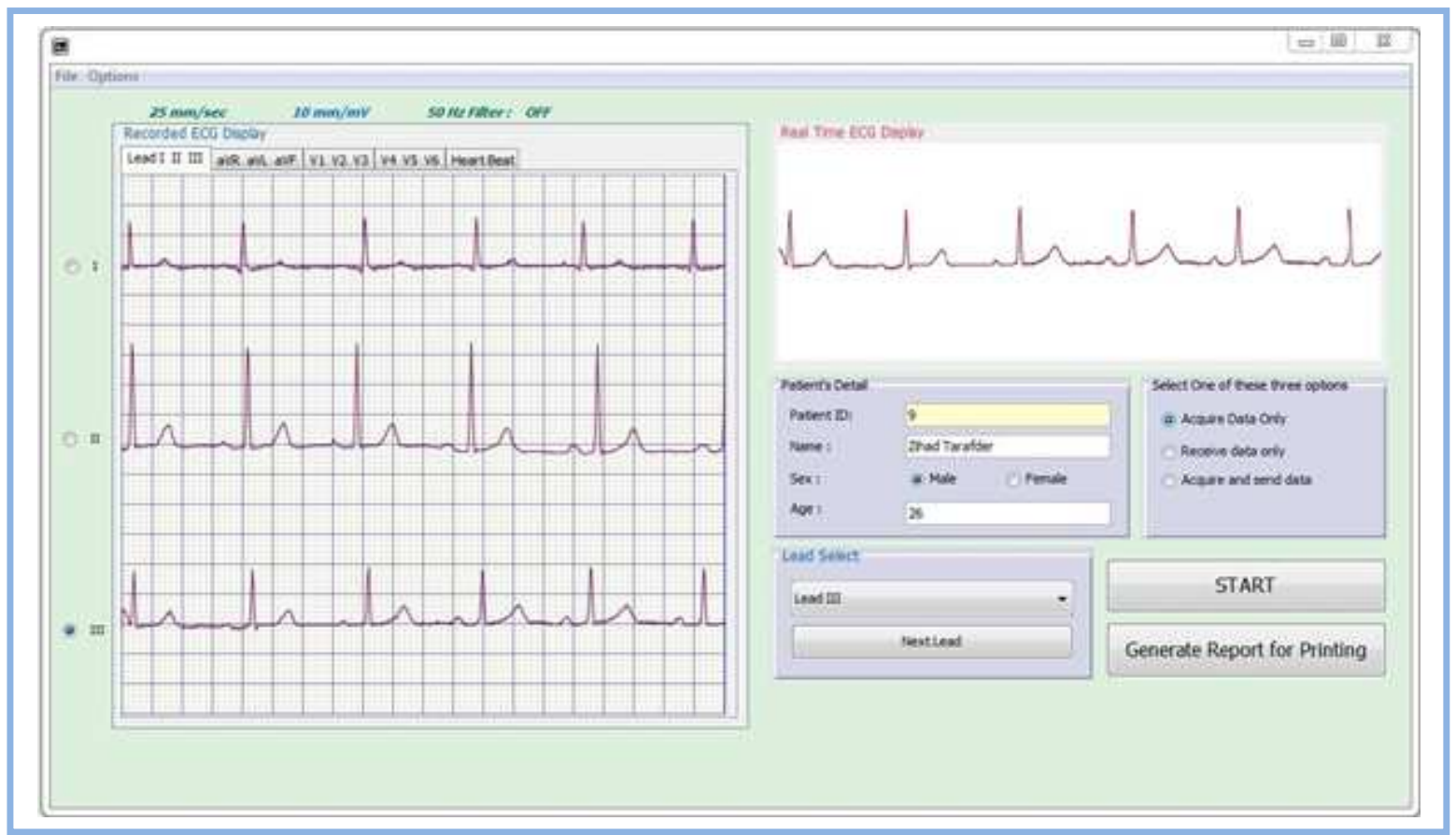

Figure 12: Software screen shot containing the ECG trace of a real subject,

\section{Discussion}

This ECG system was developed keeping the view that indigenous development of modern medical equipment will help the Third World countries to provide medical care to its people at affordable cost. For this development of indigenous capability in design and fabrication is essential. Such locally made equipment will provide service through a long period of time as local maintenance and repair will be available at low cost.

Developing a PC based ECG equipment was chosen because this will reduce much of the hardware complexity and also reduce run time printing paper cost required for storage and graphical display of data. Since low cost PC's are widely available in most of the Third World countries which can be easily maintained, and if hard copy of ECG signals is required it can be printed out by the PC. This ECG equipment has low affordable manufacturing, repair and maintenance cost, also with wide options of usability, so it will benefit the common people of the Third World both in health service and economical aspects.

This paper presents details of the design aspects and technological issues of a computer based ECG equipment as designs of such equipment are still not available for common knowledge, even after 50 years of development of 12 Lead ECG configurations. Details of patient safety consideration are also presented, which is very important in medical instrumentation since it involves human life. Some details on design considerations and patient safety are available here (Rabbani et al 2011).

This circuitry and the necessary software were developed from basic scientific knowledge. The major challenge was to develop the resistor network with analog switching for different Leads with low component count. Another challenge was to implement USB protocol for data acquisition without purchasing a commercial data acquisition system, which are expensive. It was also interesting to find 
out that even after a decade of introduction of USB with open specifications, no experience with USB

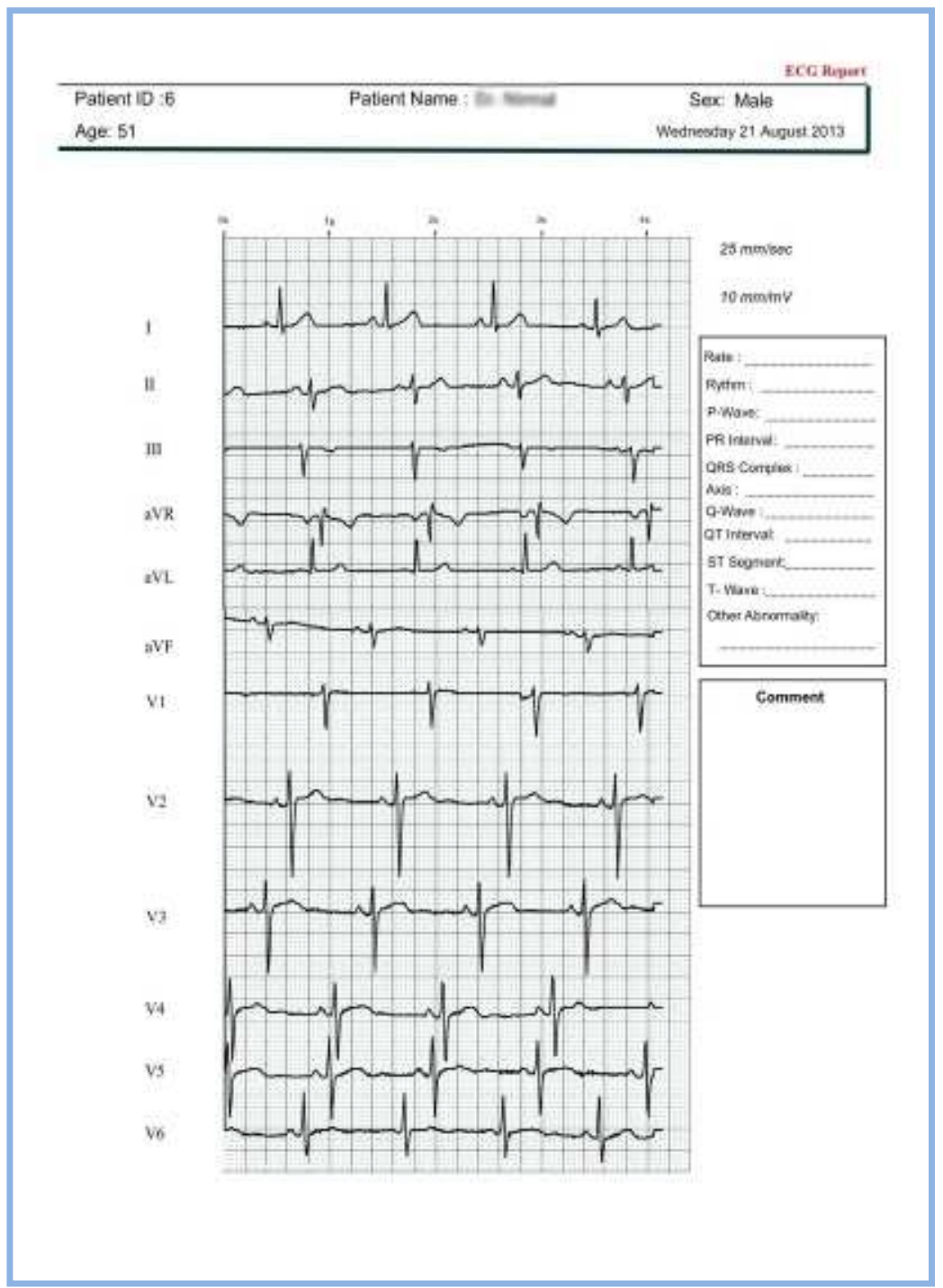

Figure 13: Generated report containing all Leads.

implementation in both software and firmware was found in Bangladesh. It points to a sign of technological disadvantage of the developing countries.

Although the design involved is complex when put together, one needs to study each individual circuitry in turn. Understanding of each segment is important to the overall success. The long experience of our team members has helped us design this system successfully. The performance of the system has been found satisfactory, however, there are rooms for improvement.

The main motivation behind this work was to empower electronics experts throughout the Third World in designing and developing modern medical equipment to an acceptable quality. The success of this work is expected to achieve this goal to a great extent. 


\section{References:}

Acharya Venkatesh 2011. Improving Common-Mode Rejection Using the Right-Leg Drive Amplifier Texas Instruments.

Atemel. 2013a. avr studio 5 [Online]. http://www.atmel.com/microsite/avr_studio_5.

Atemel. 2013b. Virtual USB port for AVR microcontrollers [Online]. http://www.obdev.at/products/vusb/index.html.

Brown BH, Smallwood RH, Barber D, Lawford PV \& Hose DR Medical physics and Physiological Measurement. 471. UK, Blackwell publications.

Brown BH, Smallwood RH, Barber DC, Lawford PV \& Hose DR Medical Physics and Biomedical Engineering. 551.

Grimnes Sverre \& Martinsen Ørjan G. 2008. Bioimpedance and Bioelectricity Basics. 341. USA, Elsevier.

Markandey Vishal 2010. ECG Implementation on the TMS320C5515 DSP Medical Development Kit (MDK). Texas Instruments.

Maryland University of. 2009. Lead Placement [Online]. Accessed 2009-08-15, http://web.archive.org/web/20110720090219/http://davidge2.umaryland.edu/ emig/ekgtu03.h $\underline{\mathrm{tml}}$.

Nottingham University of. 2009. ECG Lead Placement [Online]. Division of Nursing. Accessed 2009-08-15, http://www.nottingham.ac.uk/nursing/practice/resources/cardiology/function/bipolar_leads.ph p.

Oracle. 2013. NetBeans [Online]. https://netbeans.org/.

Rabbani KS. How to deliver the benefits of modern Biomedical Engineering to the common people in the economically developing countries. 9th Medical Engineering Conference, 1995a Yamagata, Japan.

Rabbani KS. Local development of Biomedical Technology - a must for the Third World. Regional Conference of IEEE-EMBS and 14th BMES, 1995b Delhi, India. 1.31-1.32.

Rabbani KS, Abir AR \& Bodiuzzaman AKM. 2011. Design and Development of a Low Cost Personal Computer based ECG Monitor. Bangladesh Journal of Medical Physics, 4.

WinAVR. 2013.

http://www.webring.org/l/rd?ring=avr;id=59;url=http\%3A\%2F\%2Fwinavr\%2Esourceforge\% 2Enet $\% 2 \mathrm{~F}$. 